\title{
A Stream Cipher based Bit-Level Symmetric Key Cryptographic Technique using Chen Prime Number
}

\author{
Sarbajit Manna \\ Assistant Professor \\ Ramakrishna Mission Vidyamandira \\ Belur Math, Howrah - 711202
}

\author{
Saurabh Dutta \\ Professor \\ Dr. B.C. Roy Engineering College \\ Jemua Road, Fuljhore, Durgapur - 713206
}

\begin{abstract}
In this paper, a stream cipher based symmetric key cryptographic technique based on Chen prime number has been proposed. The proposed technique is suitable for encryption and decryption of a large number of files of almost any type. A symmetric key is formed by the sender directly from the plain text using Chen prime number. Then a symmetric key value is derived from the symmetric key which is used to form the cipher text. The symmetric key is sent to the receiver by the sender. The symmetric key value is derived by the receiver from the symmetric key which is used for decryption. To enhance security, focus is to secure the symmetric key value rather than the symmetric key.
\end{abstract}

\section{Keywords}

Chen Prime Number, Stream Cipher, Symmetric Key, Plain Text, Cipher Text.

\section{INTRODUCTION}

Now-a-days the Internet has become an essential mode of communication and is being increasingly used day by day. So, the information security becomes an important issue to deal with. Some algorithms are based on symmetric key, some are based on asymmetric key, but the focus is to secure the information which is the essence of communication [7]-[9].

In this paper, a new symmetric key algorithm based on Chen prime number has been proposed, where the plain text is considered as a stream of binary bits. During encryption process, a symmetric key value is generated from the symmetric key, which is used to form the cipher text. During decryption process, the plain text can be re-generated from the cipher text using the symmetric key value. The algorithm consists of following four major components [2], [6].

\subsection{Symmetric Key Generation Plain Text $\longrightarrow$ Symmetric Key}

\subsection{Symmetric Key Value Generation Symmetric Key $\longrightarrow$ Symmetric Key Value}

\subsection{Encryption Mechanism}

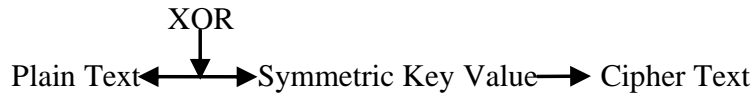

\subsection{Decryption Mechanism}

XOR

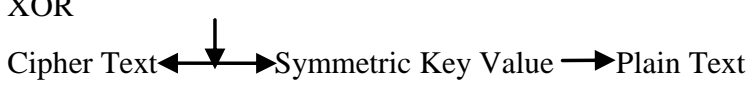

The proposed algorithm is presented in section 2. One sample implementation is shown in section 3, whereas the experimental result and analysis are shown in section 4 . Finally, section 5 draws the conclusion.

\section{PROPOSED ALGORITHM}

A prime number $p$ is called a Chen prime if $p+2$ is either a prime or a product of two primes. 31 is a Chen prime number because $31+2=33$ is a product of two prime numbers 3 and 11 . 17 is a Chen prime number because $17+2=19$ is a prime number. 43 is not a Chen prime number because $43+2=45$ is neither a prime number nor a product of two prime numbers [6].

Section 2.1 and section 2.2 respectively describe encryption and decryption mechanisms.

\subsection{Encryption Mechanism}

The encryption mechanism consists of the following five steps.



Steps are described in section 2.1.1 to section 2.1.5.

\subsubsection{Binary Stream of Bits Formation of the Input File}

The input file is read one character at a time. Each character is converted into 8-bit binary representation according to its ASCII value.

Let, the character from the input file is ' $\mathrm{d}$ '. The binary stream of bits for 'd' (ASCII value 100) will be 01100100 which is represented in an array PT of 8-bits.

\subsubsection{Symmetric Key Generation from the Plain $\operatorname{Text}(P T)$}

The size of the symmetric key is 24 bits having 4 blocks of size 8 bits, 7 bits, 1 bit and 8 bits respectively. The $1^{\text {st }}$ block represents sum of positional weights of Chen prime bit positions of the plain text $\left(\mathrm{S}_{\mathrm{CP}}\right)$. The $2^{\text {nd }}$ block represents sum of positional weights of remaining bit positions of the plain text $\left(S_{\mathrm{RP}}\right)$. The $3^{\text {rd }}$ block represents the forward $(0)$ or backward (1) direction which is derived from the expression $\left(\mathrm{ABS}\left(\mathrm{S}_{\mathrm{CP}}-\mathrm{S}_{\mathrm{RP}}\right)\right) \% 2$ where $\mathrm{ABS}$ and \% represents absolute value and modulus operation respectively. The $4^{\text {th }}$ block represents $\mathrm{S}_{\mathrm{RP}}$ th $/ \mathrm{S}_{\mathrm{CP}}{ }^{\text {th }}$ Chen prime term starting from the plain text depending on forward / backward direction respectively. If the derived value of the above expression is 0 , then 0 (represents forward direction) is stored in $3^{\text {rd }}$ block of key and the binary equivalent of $S_{R P}$ is stored in $4^{\text {th }}$ block of key. If the derived value of the above expression is 1 , then 1 (represents backward direction) is stored in $3^{\text {rd }}$ block of key and the binary equivalent of $S_{C P}$ is stored in $4^{\text {th }}$ block of key. 
The Chen Prime bit positions (CP) of a 8 -bit number are : 2, $3,5,7$ and the Remaining bit positions (RP) are : $0,1,4,6$.

So, for plain text ' $d$ ', the $\mathrm{CP}$ positions $2,3,5,7$ and RP positions are $0,1,4,6 . \mathrm{S}_{\mathrm{CP}}=4+0+32+0=36=00100100$ in binary, it is stored in $1^{\text {st }}$ block of key and $\mathrm{S}_{\mathrm{RP}}=0+0+0+64=$ $64=1000000$ in binary, it is stored in $2^{\text {nd }}$ block of key. $\left(\mathrm{ABS}\left(\mathrm{S}_{\mathrm{CP}}-\mathrm{S}_{\mathrm{RP}}\right)\right) \% 2=(\mathrm{ABS}(36-64)) \% 2=0$, it is stored in $3^{\text {rd }}$ block of key. As 0 represents Forward Direction (FD), So, 01000000 i.e. the binary equivalent of $S_{R P}$ is stored in $4^{\text {th }}$ block of key.

\subsubsection{Symmetric Key Value Generation from the}

Plain Text $(P T)$

If the value in $3^{\text {rd }}$ block of the key is 0 , then forward movement is taken and $\mathrm{S}_{\mathrm{RP}}^{\text {th }}$ Chen prime starting from $\mathrm{PT}$ is calculated. If the value in $3^{\text {rd }}$ block of the key is 1 , then backward movement is taken and $\mathrm{S}_{\mathrm{CP}}{ }^{\text {th }}$ Chen prime starting from PT is calculated. If the $\mathrm{S}_{\mathrm{CP}}{ }^{\text {th }} \mathrm{Chen}$ prime starting from PT in backward direction is negative, then the absolute value is taken.

So, for plain text 'd', as the value in $3^{\text {rd }}$ block of the key is 0 , so forward movement is taken and $64^{\text {th }}\left(\mathrm{S}_{\mathrm{RP}}^{\text {th }}\right)$ Chen prime starting from 100 (ASCII value of 'd') is calculated. It is 659 .

\subsubsection{Binary Representation of Symmetric Key value}

The symmetric key value is represented in binary form. If the number of bits in the binary form is not a multiple of 8 , then it is made multiple of 8 by inserting the remaining number of 0 's to the left side of MSB.

So, for plain text ' $d$ ', $659_{10}$ is represented in equivalent binary form. The total number of bits in binary representation of 659 is 10 , which is not a multiple of 8 . It is made multiple of 8 by inserting the remaining number of 0 's (six) to the left side of MSB. $659_{10}=1010010011_{2}=0000001010010011_{2}($ By inserting six 0 's to the left of MSB to make it a multiple of 8).

\subsubsection{Cipher Text (CT) Generation}

Cipher text is generated by successively XORing the 8-bit blocks starting from MSB of the binary form of the symmetric key value and finally with the 8-bit binary representation of the plain text.

So, for plain text ' $d$ ', Cipher Text(CT) is generated by the following procedure where $\oplus$ represents XOR operation.

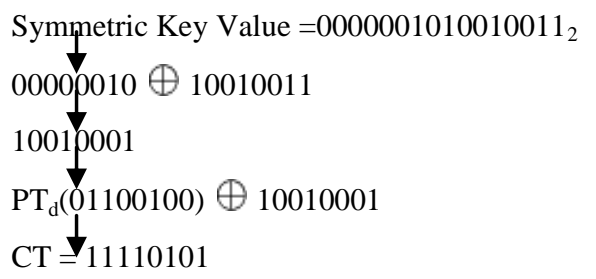

So, $\mathrm{CT}=11110101_{2}=245_{10}=\tilde{\mathrm{o}}, \mathrm{CT}$ for $\mathrm{PT}_{\mathrm{d}}$ is $\tilde{\mathrm{o}}$.

\subsection{Decryption Mechanism}

The decryption mechanism consists of the following three steps.

Conversion of Cipher Text (CT) in binary form

Formation of symmetric key value from symmetric key

Plain Text (PT) generation

Steps are described in section 2.2.1 to section 2.2.3.

\subsubsection{Conversion of Cipher Text (CT) in Binary \\ form}

The entire encrypted file is read by the receiver and each character is converted into 8-bit binary form from their ASCII values.

So, ASCII value of cipher text 'õ' is $245_{10}=11110101_{2}$ in binary.

\subsubsection{Formation of Symmetric Key Value from}

Symmetric Key

As the symmetric key is known to the receiver, so the symmetric key value is generated by the receiver from the symmetric key. The symmetric key value is then represented in binary form. If the total number of bits in the binary representation of the symmetric key is not a multiple of 8 , then it is made multiple of 8 by inserting remaining number of 0 's to the left of MSB. Otherwise it is kept as it is.

For cipher text 'õ', the receiver generates the symmetric key value $659_{10}$ and it is represented in binary form $\left(1010010011_{2}\right)$. The total number of bits in $1010010011_{2}$ is 10 , it is not a multiple of 8 . So to make it multiple of 8 , remaining 0's(6) are inserted to the left of MSB of $1010010011_{2}$. So the binary representation of $659_{10}$ becomes $0000001010010011_{2}$.

\subsubsection{Plain Text (PT) Generation}

Plain text is generated by successively XORing the 8-bit blocks starting from MSB of the binary form of the symmetric key value and finally with the 8-bit binary representation of the cipher text.

So, for cipher text 'õ', Plain Text(PT) is generated by the following procedure where $\oplus$ represents XOR operation.

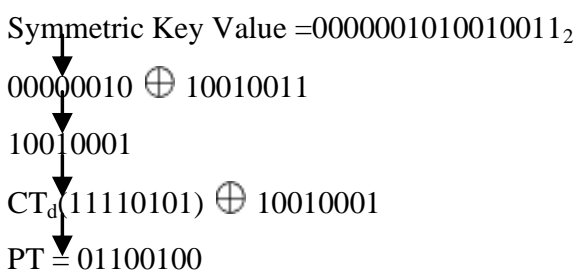

So, $\mathrm{PT}=01100100_{2}=100_{10}=\mathrm{d}$. So $\mathrm{PT}_{\mathrm{d}}$ is successfully recovered from $\mathrm{CT}_{\tilde{0}}$.

\section{IMPLEMENTATION}

Following tables contain the detailed description of the encryption and decryption process of the proposed algorithm. A plain text file of size $1 \mathrm{~KB}$ (test.txt) is taken for encryption. The content of the plain text file is "do". Then it is encrypted to form the encrypted file ct_test.txt. From the encrypted file, the decrypted file pt_test.txt is formed by the decryption process. It is seen that the contents of the plain text file and the decrypted file is same.

Table 1. Encryption Process

\begin{tabular}{|c|c|c|}
\hline Plain Text (PT) & $\mathrm{d}$ & $\mathrm{o}$ \\
\hline $\begin{array}{c}\text { ASCII value of } \\
\text { each character }\end{array}$ & 100 & 111 \\
\hline $\begin{array}{c}\text { Binary equivalent } \\
\text { of ASCII value }\end{array}$ & 01100100 & 01101111 \\
\hline $\mathbf{S}_{\mathbf{C P}}$ & $36(00100100)$ & $44(00101100)$ \\
\hline $\mathbf{S}_{\mathbf{R P}}$ & $64(1000000)$ & $67(1000011)$ \\
\hline
\end{tabular}




\begin{tabular}{|c|c|c|}
\hline $\begin{array}{c}\text { Direction } \\
\left(\mathrm{ABS}\left(\mathbf{S}_{\mathrm{CP}}-\mathbf{S}_{\mathrm{RP}}\right)\right) \% 2\end{array}$ & 0 (Forward) & 1 (Backward) \\
\hline $\mathbf{S}_{\mathrm{CP}} / \mathrm{S}_{\mathrm{RP}}$ & $\begin{array}{c}\mathrm{S}_{\mathrm{RP}}=64 \\
(1000000)\end{array}$ & $\begin{array}{c}\mathrm{S}_{\mathrm{CP}}=44 \\
(00101100)\end{array}$ \\
\hline Key Value (KV) & $\begin{array}{c}659 \\
\left(64^{\text {th }} \text { Chen prime }\right. \\
\text { from } 100 \text { in forward } \\
\text { direction })\end{array}$ & $\begin{array}{c}101 \\
\left(44^{\text {th }} \text { Chen prime }\right. \\
\text { from } 111 \text { in } \\
\text { backward } \\
\text { direction) absolute } \\
\text { value is taken }\end{array}$ \\
\hline $\begin{array}{c}\text { Binary equivalent } \\
\text { of key value }\end{array}$ & $\begin{array}{c}1010010011 \\
\text { (No. of bits not a } \\
\text { multiple of 8) }\end{array}$ & 01100101 \\
\hline $\begin{array}{l}\text { Making key value } \\
\text { multiple of } 8\end{array}$ & $\begin{array}{c}\text { Required } \\
\text { 0000001010010011 } \\
\text { (Six 0’s inserted to } \\
\text { the left) }\end{array}$ & Not required \\
\hline $\begin{array}{c}\text { Cipher text } \\
(\mathrm{KV} \oplus \mathbf{P T}) \text { in } \\
\text { binary }\end{array}$ & $\begin{array}{c}00000010 \bigoplus \\
10010011 \oplus \\
01100100= \\
11110101\end{array}$ & $\begin{array}{c}01100101 \oplus \\
01101111= \\
00001010\end{array}$ \\
\hline $\begin{array}{c}\text { Decimal equivalent } \\
\text { of cipher text }\end{array}$ & 245 & 10 \\
\hline Cipher Text (CT) & $\tilde{\mathrm{o}}$ & LF \\
\hline
\end{tabular}

Table 2. Decryption Process

\begin{tabular}{|c|c|c|}
\hline Cipher Text (CT) & $\tilde{\mathrm{o}}$ & LF \\
\hline $\begin{array}{l}\text { ASCII value of each } \\
\text { character }\end{array}$ & 245 & 10 \\
\hline $\begin{array}{c}\text { Binary equivalent of } \\
\text { ASCII value }\end{array}$ & 11110101 & 00001010 \\
\hline $\begin{array}{l}\text { Key Value generated } \\
\text { from key }(\mathrm{KV})\end{array}$ & 659 & 101 \\
\hline $\begin{array}{c}\text { Binary equivalent of } \\
\text { key value }\end{array}$ & $\begin{array}{c}1010010011 \\
\text { (No. of bits not a } \\
\text { multiple of 8) }\end{array}$ & 01100101 \\
\hline $\begin{array}{l}\text { Making key value } \\
\text { multiple of } 8\end{array}$ & $\begin{array}{c}\text { Required } \\
\text { 0000001010010011 } \\
\text { (Six 0's inserted to } \\
\text { the left) }\end{array}$ & Not required \\
\hline $\begin{array}{c}\text { Plain text } \\
(\mathbf{K V} \oplus \mathbf{C T}) \text { in binary }\end{array}$ & $\begin{array}{c}00000010 \bigoplus \\
10010011 \oplus \\
11110101= \\
01100100\end{array}$ & $\begin{array}{c}01100101 \oplus \\
00001010= \\
01101111\end{array}$ \\
\hline $\begin{array}{c}\text { Decimal equivalent of } \\
\text { cipher text }\end{array}$ & 100 & 111 \\
\hline Plain Text (PT) & $\mathrm{d}$ & $\mathrm{O}$ \\
\hline
\end{tabular}

\section{RESULT AND ANALYSIS}

This section presents results for files of different categories namely, .EXE, .DLL, .COM, .SYS, and .TXT. Each file category contains ten different files. Each section consists of tables with information on source file size, target file size, encryption time, chi square value with the degree of freedom and avalanche percentage. Implementation of the algorithm and different types of analysis has been done using $\mathrm{C}$ on a computer with Intel Pentium IV $2.40 \mathrm{GHz}$ processor having 512 MB RAM.

The Pearson's chi-squared test has been performed here between the source and the encrypted files to test the nonhomogeneity of the two files which means whether the observations onto encrypted files are in good agreement with a hypothetical distribution. In this case, the chi square distribution is being performed with $(256-1)=255$ degrees of freedom, 256 being the total number of classes of possible characters in the source as well as in the encrypted files. If the observed value of the statistic exceeds the tabulated value at a given level, the null hypothesis is rejected [1]

The "Pearson's chi-squared" or the "Goodness-of-fit chisquare" is defined by the following equation:

$\mathrm{X}^{2}=\Sigma\left\{\left(f_{0}-f_{\mathrm{e}}\right)^{2} / f_{\mathrm{e}}\right\}$

Here $f_{\mathrm{e}}$ and $f_{0}$ respectively stand for the frequency of a character in the source file and that of the same character in the corresponding encrypted file. On the basis of this formula, the chi-square values have been calculated for sample pairs of source and encrypted files [1].

The avalanche effect is a very important property of cryptographic algorithms. The avalanche effect is evident if, when an input is changed slightly, the output changes drastically. If a bit in the plain text is flipped or changed, then for an evident avalanche effect, almost half of the cipher text bits are changed in the cipher text. The small change can occur either in the plaintext or in the key so that it can cause a drastic change in the cipher text. In the proposed technique, the $3^{\text {rd }}$ bit of each plaintext is flipped. The key for each plaintext changes automatically with the flipping of bits in the plaintext. The avalanche percentage for each file is shown in following tables [3]-[5].

Section 4.1, section 4.2, section 4.3, section 4.4 and section 4.5 respectively describe the result for .EXE, .DLL, .COM, .SYS, .TXT files.

\subsection{Result for .EXE files}

Table 3 presents the result for ten .EXE files. The file sizes are in the range of 5632 bytes to 21811 bytes. Encrypted file size as well as decrypted file size is same as the source file. The encryption time ranges between 94.53 seconds to 397.82 seconds. The chi square value for each of the cases lies in the range of 14092 to 60098 with the degree of freedom ranging from 209 to 255 . The achieved avalanche ranges from $15.37 \%$ to $40.56 \%$.

Table 3. Result For .EXE Files

\begin{tabular}{|c|c|c|c|c|}
\hline $\begin{array}{c}\text { Source/ } \\
\text { Target } \\
\text { File Size } \\
\text { (Byte) }\end{array}$ & $\begin{array}{c}\text { Encryption } \\
\text { Time (S) }\end{array}$ & $\begin{array}{c}\text { Chi } \\
\text { Square } \\
\text { Value }\end{array}$ & $\begin{array}{c}\text { Degree } \\
\text { of } \\
\text { Freedom }\end{array}$ & $\begin{array}{c}\text { Avalanche } \\
\text { Achieved } \\
(\%)\end{array}$ \\
\hline 12288 & 208.56 & 14092 & 209 & 15.37 \\
\hline 11776 & 214.48 & 55873 & 252 & 31.19 \\
\hline 11264 & 197.40 & 60098 & 252 & 40.56 \\
\hline 8192 & 140.66 & 38470 & 253 & 27.68 \\
\hline
\end{tabular}




\begin{tabular}{|c|c|c|c|c|}
\hline 5632 & 94.53 & 22748 & 240 & 24.21 \\
\hline 7680 & 135.56 & 34705 & 245 & 29.06 \\
\hline 18432 & 340.09 & 23091 & 255 & 32.00 \\
\hline 15360 & 268.31 & 46243 & 254 & 27.26 \\
\hline 21811 & 397.82 & 20561 & 255 & 30.35 \\
\hline 12288 & 202.12 & 22154 & 223 & 15.98 \\
\hline
\end{tabular}

Fig. 1 graphically compares the encryption time with the source file size for .EXE files. Each horizontal bar of color gray stands for the source file size in KB and the horizontal bar of color blackstands for the encryption time in second. It is observed from the figure that encryption time is directly proportional to the source file size.



Fig. 1: A comparison between source file size with encryption time for .EXE files

\subsection{Result for .DLL files}

Table 4 presents the result for ten .DLL files. The file sizes are in the range of 10752 bytes to 34816 bytes. Encrypted file size as well as decrypted file size is same as the source file. The encryption time ranges between 111.39 seconds to 522.45 seconds. The chi square value for each of the cases lies in the range of 15708 to 86039 with the degree of freedom ranging from 187 to 255 . The achieved avalanche ranges from $20.55 \%$ to $31.61 \%$.

Table 4. Result For .DLL Files

\begin{tabular}{|c|c|c|c|c|}
\hline $\begin{array}{c}\text { Source/ } \\
\text { Target } \\
\text { File Size } \\
\text { (Byte) }\end{array}$ & $\begin{array}{c}\text { Encryption } \\
\text { Time (S) }\end{array}$ & $\begin{array}{c}\text { Chi } \\
\text { Square } \\
\text { Value }\end{array}$ & $\begin{array}{c}\text { Degree } \\
\text { of } \\
\text { Freedom }\end{array}$ & $\begin{array}{c}\text { Avalanche } \\
\text { Achieved } \\
(\boldsymbol{\%})\end{array}$ \\
\hline 17408 & 274.84 & 27692 & 255 & 30.22 \\
\hline 17408 & 111.39 & 30629 & 187 & 20.55 \\
\hline 13312 & 187.68 & 45919 & 253 & 23.31 \\
\hline 30208 & 464.07 & 55796 & 255 & 31.61 \\
\hline 34816 & 522.45 & 15708 & 255 & 28.53 \\
\hline 11264 & 162.30 & 29011 & 253 & 28.80 \\
\hline 16896 & 235.69 & 86039 & 248 & 29.80 \\
\hline 10752 & 153.62 & 62629 & 253 & 27.23 \\
\hline 15872 & 237.33 & 47901 & 255 & 30.07 \\
\hline 13312 & 193.67 & 62594 & 255 & 29.08 \\
\hline
\end{tabular}

Fig. 2 graphically compares the encryption time with the source file size for .DLL files. Each horizontal bar of color gray stands for the source file size in $\mathrm{KB}$ and the horizontal bar of color black stands for the encryption time in second. It is observed from the figure that encryption time is directly proportional to the source file size.

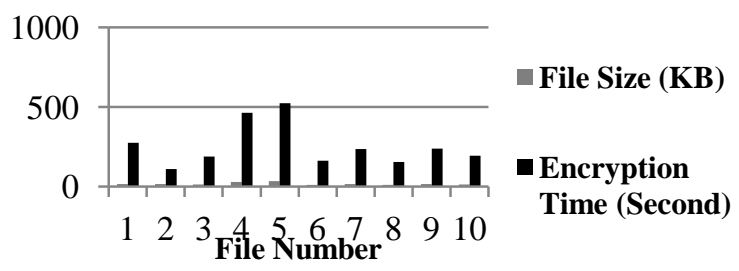

Fig. 2: A comparison between source file size with encryption time for .DLL files

\subsection{Result for .COM files}

Table 5 presents the result for ten .COM files. The file sizes are in the range of 1126 bytes to 69836 bytes. Encrypted file size as well as decrypted file size is same as the source file. The encryption time ranges between 16.86 seconds to 1276.25 seconds. The chi square value for each of the cases lies in the range of 976 to 51245 with the degree of freedom ranging from 186 to 255 . The achieved avalanche ranges from $25.31 \%$ to $34.71 \%$.

Table 5. Result For .COM Files

\begin{tabular}{|c|c|c|c|c|}
\hline $\begin{array}{c}\text { Source/ } \\
\text { Target } \\
\text { File Size } \\
\text { (Byte) }\end{array}$ & $\begin{array}{c}\text { Encryption } \\
\text { Time (S) }\end{array}$ & $\begin{array}{c}\text { Chi } \\
\text { Square } \\
\text { Value }\end{array}$ & $\begin{array}{c}\text { Degree } \\
\text { of } \\
\text { Freedom }\end{array}$ & $\begin{array}{c}\text { Avalanche } \\
\text { Achieved } \\
(\%)\end{array}$ \\
\hline 7680 & 134.41 & 27141 & 250 & 27.73 \\
\hline 9216 & 159.12 & 29518 & 251 & 30.21 \\
\hline 7168 & 122.10 & 49458 & 243 & 28.04 \\
\hline 69836 & 1276.25 & 15219 & 255 & 30.01 \\
\hline 29696 & 565.52 & 11644 & 255 & 34.71 \\
\hline 26112 & 491.09 & 35335 & 255 & 28.47 \\
\hline 19660 & 352.90 & 51245 & 254 & 28.00 \\
\hline 7014 & 126.05 & 32029 & 255 & 30.57 \\
\hline 14643 & 260.90 & 32093 & 255 & 28.49 \\
\hline 1126 & 16.86 & 976 & 186 & 25.31 \\
\hline
\end{tabular}

Fig. 3 graphically compares the encryption time with the source file size for .COM files. Each horizontal bar of color gray stands for the source file size in $\mathrm{KB}$ and the horizontal bar of color black stands for the encryption time in second. It is observed from the figure that encryption time is directly proportional to the source file size.

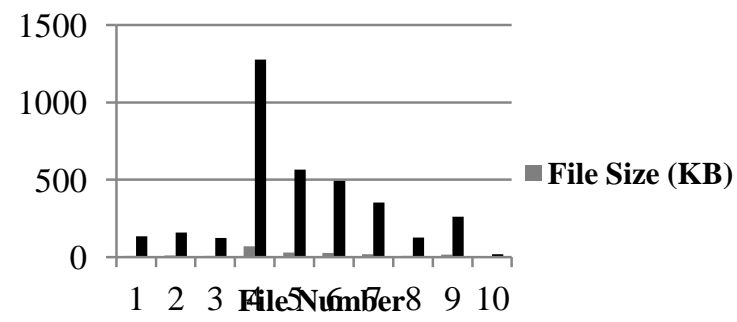

Fig. 3: A comparison between source file size with encryption time for .COM files

\subsection{Result for .SYS files}

Table 6 presents the result for ten .SYS files. The file sizes are in the range of 4218 bytes to 17612 bytes. Encrypted file size as well as decrypted file size is same as the source file. The encryption time ranges between 57.61 seconds to 257.21 seconds. The chi square value for each of the cases lies in the range of 9837 to 70763 with the degree of freedom ranging from 229 to 255 . The achieved avalanche ranges from $20.98 \%$ to $29.65 \%$.

Table 6. Result For .SYS Files

\begin{tabular}{|c|c|c|c|c|}
\hline $\begin{array}{c}\text { Source/ } \\
\text { Target } \\
\text { File Size } \\
\text { (Byte) }\end{array}$ & $\begin{array}{c}\text { Encryption } \\
\text { Time (S) }\end{array}$ & $\begin{array}{c}\text { Chi } \\
\text { Square } \\
\text { Value }\end{array}$ & $\begin{array}{c}\text { Degree } \\
\text { of } \\
\text { Freedom }\end{array}$ & $\begin{array}{c}\text { Avalanche } \\
\text { Achieved } \\
(\%)\end{array}$ \\
\hline 10240 & 147.80 & 47769 & 254 & 28.01 \\
\hline 11264 & 161.92 & 47556 & 252 & 26.92 \\
\hline 15872 & 237.17 & 70088 & 255 & 28.67 \\
\hline 10240 & 155.66 & 36554 & 252 & 27.85 \\
\hline 10240 & 147.31 & 56506 & 252 & 28.09 \\
\hline
\end{tabular}




\begin{tabular}{|c|c|c|c|c|}
\hline 9021 & 132.70 & 48945 & 252 & 28.87 \\
\hline 4218 & 57.61 & 9837 & 229 & 24.20 \\
\hline 16384 & 232.22 & 44655 & 252 & 20.98 \\
\hline 4761 & 71.95 & 37768 & 249 & 29.65 \\
\hline 17612 & 257.21 & 70763 & 255 & 28.69 \\
\hline
\end{tabular}

Fig. 4 graphically compares the encryption time with the source file size for .SYS files. Each horizontal bar of color gray stands for the source file size in $\mathrm{KB}$ and the horizontal bar of color black stands for the encryption time in second. It is observed from the figure that encryption time is directly proportional to the source file size.

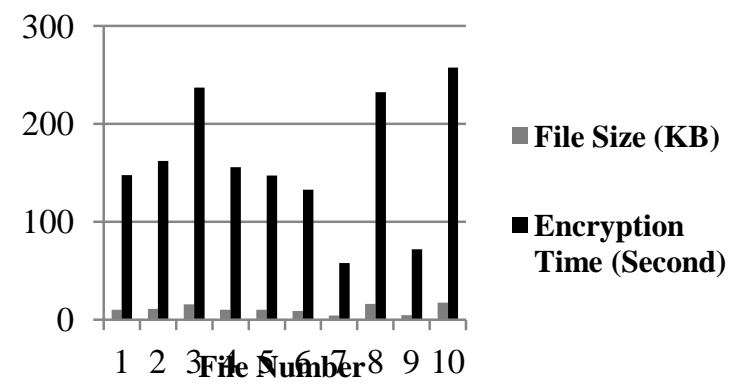

Fig. 4: A comparison between source file size with encryption time for .SYS files

\subsection{Result for .TXT files}

Table 7 presents the result for ten .TXT files. The file sizes are in the range of 133 bytes to 32563 bytes. Encrypted file size as well as decrypted file size is same as the source file. The encryption time ranges between 0.77 seconds to 155.60 seconds. The chi square value for each of the cases lies in the range of -161 to 16105 with the degree of freedom ranging from 53 to 134 . The achieved avalanche ranges from $23.79 \%$ to $33.47 \%$.

Table 7. Result For .TXT Files

\begin{tabular}{|c|c|c|c|c|}
\hline $\begin{array}{c}\text { Source/ } \\
\text { Target } \\
\text { File Size } \\
\text { (Byte) }\end{array}$ & $\begin{array}{c}\text { Encryption } \\
\text { Time (S) }\end{array}$ & $\begin{array}{c}\text { Chi } \\
\text { Square } \\
\text { Value }\end{array}$ & $\begin{array}{c}\text { Degree } \\
\text { of } \\
\text { Freedom }\end{array}$ & $\begin{array}{c}\text { Avalanche } \\
\text { Achieved } \\
(\%)\end{array}$ \\
\hline 1331 & 7.42 & 3994 & 101 & 33.47 \\
\hline 5416 & 31.91 & 10536 & 114 & 29.87 \\
\hline 6451 & 35.86 & 16105 & 108 & 25.16 \\
\hline 133 & 0.77 & 253 & 53 & 29.89 \\
\hline 829 & 4.34 & 1032 & 88 & 32.70 \\
\hline 22937 & 115.34 & 6958 & 121 & 26.82 \\
\hline 2201 & 11.70 & 4099 & 104 & 28.64 \\
\hline 2498 & 13.52 & 5120 & 103 & 31.40 \\
\hline 32563 & 155.60 & -161 & 94 & 23.79 \\
\hline 19046 & 101.50 & 10177 & 134 & 30.62 \\
\hline
\end{tabular}

Fig. 5 graphically compares the encryption time with the source file size for .TXT files. Each horizontal bar of color gray stands for the source file size in KB and the horizontal bar of color black stands for the encryption time in second. It is observed from the figure that encryption time is directly proportional to the source file size.

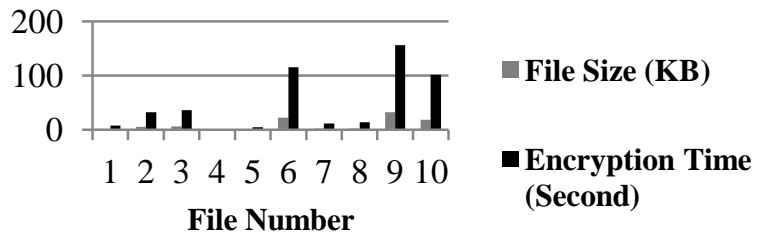

Fig. 5: A comparison between source file size with encryption time for .TXT files

\section{CONCLUSION}

The proposed symmetric-key algorithm has been developed keeping security factor in mind. In the proposed algorithm, the generated symmetric key is totally based on the plain text and Chen prime number. As the plain text changes, the symmetric key also changes automatically. The symmetric key value generated from the symmetric key gives an extra edge in security for the algorithm. The key space is directly proportional to the source file size. The proposed technique presented in this paper is simple to implement using any popular high level language. The size of the encrypted file and decrypted file is same as the source file size. So any additional memory is not needed to store the encrypted and decrypted file. The execution time is dependent on the source file size, not on the type of the file as the encryption and decryption has been done in bit level. For most of the files, the achieved avalanche percentage is between 25 and 35 . The avalanche percentage will be better if more than one bit is flipped. The avalanche effect can also be calculated not only flipping one or more bits of the plain text, but also flipping any number of bits of the symmetric key or even both the plain text and the symmetric key. Not only XOR operation but also any one or more reversible logical operations can be applied on the plaintext to get the cipher text.

\section{REFERENCES}

[1] Saurabh Dutta, "An Approach towards Development of Efficient Encryption Technique", Ph.D. Thesis, The University of North Bengal, 2004.

[2] Ramkrishna Das, Saurabh Dutta, "An Approach of Bitlevel Private-key Encryption Scheme based on Armstrong Number and Perfect Number in Selective Mode", Bulletin \& Engineering Science (BES), ISSN : 0974- 7176 Vol. 5 No. 1.

[3] http://en.wikipedia.org/wiki/Avalanche_effect

[4] SriramRamanujam and MarimuthuKaruppiah "Designing an algorithm with high Avalanche Effect" IJCSNS International Journal of Computer Science and Network Security, VOL.11 No.1, January 2011.

[5] Manas Paul, Tanmay Bhattacharya, Suvajit Pal, RanitSaha, "A Novel Generic Session Based Bit Level Encryption Technique to Enhance Information Security", (IJCSIS) International Journal of Computer Science and Information Security, Vol. 3, No. 1, 2009.

[6] http://en.m.wikipedia.org/wiki/Chen_prime

[7] Mark Nelson, Jean-Loup Gailly, The Data Compression Book. BPB Publication

[8] Atul Kahate, Cryptography and Network security, Tata McGraw Hill Publishing Company Limited.

[9] William Stallings, Cryptography and Network security: Principles and Practice (Second Edition), Pearson Education Asia, Sixth Indian Reprint 2002. 